\title{
Alterations of the B-Cell Response by HIV-1 Replication
}

\author{
Xiaoying Shen and \\ Department of Surgery, Duke Human Vaccine Institute, Duke University Medical Center, Rm \\ 4030 MSRBII, 2 Genome Ct., Durham, NC 27710, USA
}

\section{Georgia D. Tomaras}

Departments of Surgery, Immunology, and Molecular Genetics and Microbiology, Duke Human

Vaccine Institute, Duke University Medical Center, Rm 4079 MSRBII, 2 Genome Ct., Durham, NC 27710, USA

Georgia D. Tomaras: gdt@duke.edu

\begin{abstract}
While the hallmark of HIV-1 infection is the progressive depletion of CD4 $4^{+} \mathrm{T}$ cells, extensive Bcell dysfunction ensues that impairs the quality of the humoral response. HIV-1 infection causes hypergammaglobulinemia, polyclonal activation, loss of memory B-cell subsets, B-cell exhaustion, aberrant B-cell surface markers, and impaired humoral responses against infections and vaccinations. The totality of the mechanisms that contribute to B-cell dysfunction in vivo is unknown, although roles for HIV proteins (Env, Tat, and Nef) and virions binding to CD21 on B cells have been identified. Recent studies suggest that early antiretroviral therapy, that minimizes virus replication, can profoundly preserve the early B-cell response to HIV-1. Thus, it is clear that there is an intricate interplay between HIV replication and stimulation of the host B-cell response to infection. A better understanding of how HIV-1 subverts a productive B-cell response is needed to inform vaccine strategies that aim to elicit long-lived plasma cells and memory B-cell responses that can act quickly upon antigen stimulation.
\end{abstract}

\section{Keywords}

HIV-1; B cell; Antibody; Humoral immunity

\section{Introduction}

The development of an effective B-cell response to HIV-1 infection is significantly and directly impaired by HIV-1 replication. During acute infection, HIV-1 is responsible for polyclonal B-cell activation, altered regulation of antibody class switching, damage to the Bcell microgenerative environments, and perturbation of memory and naïve B-cell subsets (Fig. 1). Preservation of some B-cell responses can be achieved by early therapeutic intervention, although long-term gains in immunological function will need to be examined. Additionally, detailed characterization of the B-cell response to HIV-1 vaccination will allow an increased understanding of the impact of HIV-1 antigen stimulation. The number and magnitude of B-cell alterations by HIV-1 transmission have a profound impact on the ability of the immune system to develop an effective B-cell response to infection.

(C) Springer Science+Business Media, LLC 2010

Correspondence to: Georgia D. Tomaras, gdt@duke.edu.

Disclosure No potential conflicts of interest relevant to this article were reported. 


\section{B-Cell Activation}

Polyclonal B-cell activation by HIV-1 was among the earliest discoveries of how HIV-1 dysregulates the initial immune response [1]. Multiple mechanisms have been identified for this B-cell pathology. Complement proteins bound to HIV virions can interact with CD21 expressed on B cells and stimulate B-cell activation [2]. In acute HIV-1 infection, there is polyclonal activation and terminal differentiation of B cells in blood and in gut-associated lymphoid tissue (GALT) B cells [3]. Furthermore, due to increased levels of numerous serum cytokines, there are multiple pathways by which B cells can be hyperactivated in HIV-1 infection. In particular, Nef was found to stimulate polyclonal activation indirectly by inducing proinflammatory cytokine production by macrophages [4]. In the non-human primate model, CD95-expressing B cells increased immediately following SIV infection and this B-cell activation was persistent during the acute phase of infection [5].

\section{Regulation of Antibody Class Switching}

Another B-cell dysfunction associated with HIV-1 infection is immunoglobulin class switching [6,7]. Cagigi et al. [8] showed that the expression of activation-induced cytidine deaminase (AID), which is required for class switch recombination and somatic hypermutation of B cells, was elevated in peripheral B cells from HIV-1 chronically infected subjects compared to that of healthy donors. Furthermore, they described higher percentages of CD27-IgA+ and CD27-IgG+ B cells in HIV-1-infected subjects [8]. These CD27- B cells can produce class-switched and somatically hypermutated antibodies. Moreover, there were higher levels of $\operatorname{IgA}$ in culture medium of ex vivo peripheral blood mononuclear cells obtained from HIV-1-infected patients compared to that of healthy donors, whereas IgG levels in the culture were comparable to that of the healthy donors [8]. HIV-1 Nef also has been shown to perturb B-cell class switching $[9,10 \bullet \bullet, 11]$. Nef suppresses CD40-dependent immunoglobulin class switching in bystander B cells [12]. It was later discovered that HIV-1 Env can bind to B cells expressing C-type lectin receptors and trigger polyclonal Ig class switch recombination through a CD40-independent pathway involving BAFF [13]. A study by Swingler and colleagues [9] demonstrated that soluble factors released by Nefproducing macrophages promoted B-cell activation and differentiation. In this study, culture supernatants from either HIV-1-infected macrophages or macrophages expressing Nef were shown to selectively stimulate the proliferation of $\mathrm{B}$ cells but not $\mathrm{T}$ cells, and to promote differentiation of the resting B cells to antibody-producing plasma cells. This stimulation of B-cell replication and differentiation was mediated by ferritin, which was shown to be induced by Nef via NF- $\mathrm{BB}$ activation. Immunodepletion of ferritin from the culture supernatant of Nef-expressing macrophages abrogated the B-cell stimulation activity of the supernatant. Building upon this work, Xu and colleagues [10••] demonstrated that Nef can be transferred from HIV-1-infected macrophages to B-cell germinal centers via intercellular conduits protruding from the macrophages. The effect on the B cells was to impair class switching to $\operatorname{IgG} 2$ and $\operatorname{IgA}$-producing plasma cells. Although this may explain the lack of a very robust IgA response to HIV-1 infection, it is clear that there are HIV-1-specific IgA responses in acute infection both systemically [3] and at mucosal sites [14].

HIV-1 virions incorporate host molecules upon budding from the host cell membrane, and these molecules also may influence HIV-1 pathogenesis. Host cell derived CD40 ligand (CD40L) that are incorporated into HIV-1 virions are capable of binding to CD40 expressed on B cells and promoting class switch [15]. In a recent study, Epeldegui et al. [16] investigated binding of host CD40L in HIV-1 virions to CD40 on B-cell surface and the effect on expression of AID in B cells. They demonstrated that HIV-1 virions produced in 293 T cells expressing CD40L, but not virions produced in $293 \mathrm{~T}$ cells without CD40L expression, stimulated AID transcription, resulting in elevated AID protein levels. This 
function was found to be dependent on CD40L:CD40 interaction, since addition of a blocking antibody to CD40L abrogated the AID expression activation. Moreover, this activation of AID expression of B cells required HIV-1 virions, since exposure of B cells to either 293 T cells that were transfected with CD40L, or the culture supernatant of the transfected cells, failed to induce AID expression.

\section{HIV-Specific B-Cell Maturation}

An effective anti-HIV antibody response may require extensive antibody maturation to derive antibodies with the breadth and potency that is required to control HIV-1 replication. All of the known broadly neutralizing monoclonal antibodies, such as 2F5, 4E10, 2G12, 1b12, VRC01, HJ16, PG9, and PG16 mAbs contain high levels of mutation their [17], suggesting extensive affinity maturation. Furthermore, HIV-1 Env trimer-binding antibodies, which are not broadly neutralizing, isolated from long-term non-progressors also showed a greater level of maturation than randomly selected B cells or other virus-specific B cells [18]. In addition, clonal populations of HIV-specific Abs were also observed in HIV-1infected patients in multiple studies [18-21]. Clonal expansion was also observed for B cells specific for influenza after a booster vaccination [22].

In a study characterizing somatic mutations in $\mathrm{V}$ and $\mathrm{J}$ regions of IgG [23], broadly neutralizing antibodies (bnAbs) b12 and 2G12 were showed to contain 44 and 51 somatic mutations in the $\mathrm{V}$ and $\mathrm{J}$ regions, respectively, whereas the average number of somatic mutations observed in those regions for non-broadly neutralizing antibodies against gp120 was 22. Somatic mutation rates for the newly described potent bnAb VRC01 are $32 \%$ for $\mathrm{V}_{\mathrm{H}}$ and $17-19 \%$ for the $\mathrm{V} \kappa[21]$. Similarly, $17.5 \%$ and $14.5 \%$ of the $\mathrm{V}_{\mathrm{H}}$ and $\mathrm{V} \kappa$, respectively, of PG9 are mutated; and $17.5 \%$ and $20.9 \%$ of the $\mathrm{VH}$ and $\mathrm{V} \kappa$, respectively, of PG16 are mutated [24].

Through the study of modified antibodies containing different levels of affinity maturation reversion mutations, the level of affinity maturation of VRC01 [25], PG9, and PG16 [24] was found to correlate with antibody neutralization breadth. It has been shown that the putative germline precursors of bnAbs against HIV-1 do not bind with significant affinity to Env or neutralize HIV-1 [24-26]. One hypothesis, therefore, of why bnAbs against HIV-1 are not commonly developed is that germline B-cell receptors (BCRs) that are capable of binding to conserved, functionally important regions of HIV-1 with an affinity above the "threshold" required for triggering and maintaining of affinity maturation toward generation of bnAbs are absent [27]. The VRC01 VH- only revertant or the Vא-only revertant bound with higher affinity to a stabilized gp120 core than the unmodified gp120. This led to the hypothesis that it is possible to initiate and guide the affinity maturation toward the generation of potent bnAbs like VRC011 by using structure-based optimization to raise the affinity of immunogens for germline B cells. [25].

Many bnAb have been shown to contain long, heavy-chain complementarity determining region 3 (HCDR3) loops [17, 28-30], raising the question of whether long HCDR3 is required for neutralization breath against HIV-1. However, VRC01 does not have long CDRH3 region (containing only 14 amino acids) [21] and still shows potent neutralization of a wide spectrum of HIV-1 isolates, indicating long HCDR3 is not necessary for bnAbls against HIV-1.

Further studies are needed as to the level of HIV-1-specific antibody maturation that occurs following HIV-1 transmission to contrast with infections that are controlled by the B-cell response. The impact of HIV-1 replication on the timing and the level of antibody affinity maturation for specific HIV-1 epitopes remains to be determined. It is plausible that the 
early impairments in the B-cell response may slow down or misdirect affinity maturation of more functional antibody responses.

\section{Effects on Microgenerative Environment}

\section{Follicular Damage and Germinal Center Loss}

More than half of the germinal centers in Peyer's patches and isolated lymphoid nodules of the terminal ileum are lost during acute HIV-1 infection, and $88 \%$ of follicles exhibited B or $\mathrm{T}$ cell apoptosis and follicular lysis [3]. Progressive depletion of proliferating B cells and disruption of the follicular dendritic cell network in germinal centers was also reported in acute SIV infection of macaques [31]. Damage to the physical location, where antibody responses to a transmitted pathogen are generated, results in profound impairment of an early and effective B-cell response. This raises the question of whether protection of germinal centers from destruction early in acute infection could help with an effective B-cell response. Studies from early treatment with antiretroviral therapy (ART) may provide answers to this question.

\section{Effects of Therapy on the B-Cell Response}

Since the B-cell abnormalities associated with HIV infection depend on virus replication, ART can have a substantial impact on minimizing the observed B-cell defects. However, studies have shown that the B-cell response does not completely recover and there are differences in acute and chronic infection with ART.

\section{ART and Acute Infection}

A recent study comparing antiretroviral treatment of patients treated early in infection compared to chronic stages found that the percentage and functional profile of resting memory B cells was higher in those treated early compared to those treated in chronic stages [34••]. Furthermore, memory B-cell responses to vaccine antigens were also preserved in vertically infected children who receive early HAART [37•]. Therapeutic intervention may serve to decrease virus replication to an extent that allows better stimulation of more functional B-cell responses. In that regard, neutralizing antibodies present at the time of challenge, in infant macaques, but in insufficient quantities to provide sterilizing protection, were found to decrease virus replication and augment B-cell responses [38••]. Thus, early intervention may be beneficial in preserving a functional B-cell response in HIV-1-infected subjects. However, further work is needed to understand the extent and duration of the Bcell preservation by early treatment.

\section{ART and Chronic Infection}

ART can control viral replication and restore $\mathrm{CD} 4^{+} \mathrm{T}$-cell counts, but fails to reverse HIVspecific memory responses, indicating that HIV-specific B-cell loss is determined by early HIV-1 infection events $[8,32,33]$. One study compared proportions of influenza nucleoprotein-specific, tetanus toxoid-specific, and HIV Env- and Gag-specific memory B cells in healthy donors and in HIV-1-infected patients at early or late stages of progression and with or without ART. Results from this study revealed that while loss of antigenspecific memory B cells is not restricted to HIV, HAART therapy was able to restore influenza nucleoprotein-specific and tetanus toxoid-specific memory B-cell populations but not HIV-specific memory B-cell populations. ART can normalize proportions of immature/ transitional cells, but decreased frequencies of IgM memory B cells persist [34••, 35, 36]. 


\section{Impact on Naïve and Memory B-Cell Populations}

Loss of memory B cells and the impact on the humoral response to HIV-1 infection has been well documented [39, 40]. Studies of SIV-infected macaques have also demonstrated a dramatic loss of B cells during the acute infection stage [5, 41, 42]. Acute SIV infection results in a decrease in SIgG-expressing memory (SIgD-CD27+) B cells in spleen and lymph nodes as well as defects in B-cell trafficking. Of note, B-cell apoptosis occurred at the time of first antibody appearance and the detection of IgG-expressing plasma cells in germinal centers [43]. Kuhrt et al. [5] demonstrated that the memory B-cell population, especially the IgD- (IgG/IgA-secreting) memory B cells, showed a faster and better recovery than the naïve B-cell population after the initial depletion of both subsets. This study highlights that infection has a detrimental effect on both the naïve and memory B-cell population that likely contributes to impaired maturation of an effective antibody response to a rapidly evolving virus infection. Moreover, the differential kinetics of the recovery of the naïve and memory populations point to a need for further study on how these B-cell defects affect the quality of the humoral response in terms of the development of broadly neutralizing antibody specificities.

\section{Frequency and Phenotype of the HIV-1 B-Cell Response}

The phenotype of B cells in HIV-1 infection has been characterized as activated, exhausted, and terminally differentiated [44-46]. Additionally, there is an increased number of immature/transitional B cells with a reduced number of CD27+ memory B cells [36, 40, 47]. Tissue-like memory B cells (properties of exhausted cells that have decreased proliferation potential) CD28-CD20hiCD21lo were increased in chronic infections [46]. Altered chemotactic ability (CXCR5/CXCCL3) was also observed during chronic HIV-1 infections [48].

In a detailed study screening $113 \mathrm{HIV}$-infected patients of various clinical statuses, $0.0031 \%$ of B cells and $0.047 \%$ of plasmablasts were found to secrete Env-specific IgGs when measured by an enzyme-linked immunospot (ELI-Spot) assay [49]. In the same study, Envspecific B cells were found to be $0.09 \%$ of total B cells when gp140 labeling was used instead of ELISpot assay. Furthermore, 64\% of the gp140-labeled B cells are CD27+, and $0.17 \%$ of all CD19+ CD27+ cells were positive for gp140 labeling. Forty-eight percent of gp140-labeled B cells expressed surface IgG, and 25\% expressed surface IgM. These data indicated that the majority of the gp140-labeled B cells were class-switched memory B cells. In addition, $0.05 \%$ of the total plasmablasts were stained positive with gp140, whereas $1.8 \%$ of the gp140-labeled B cells were plasmablasts. The phenotypic profile of B cells from different stages of HIV-1 infection and post-vaccination provides a window into the functional status of the B-cell response.

\section{HIV-1 Env Vaccination and B-Cell Response}

Can HIV-1 vaccination induce improved B-cell responses, compared to natural HIV-1 infection, due to the absence of damage to B-cell microgenerative environments and no loss of $\mathrm{CD} 4^{+} \mathrm{T}$-cell help that characterizes a replicating infection? Although vaccination of HIV-1-uninfected individuals can likely stimulate HIV-1-specific antigen responses without negatively perturbing the B-cell response, other obstacles remain. Foremost is the appropriate presentation of the most vulnerable epitopes within the immunogen. Another challenge is to provide for a way to drive robust affinity maturation. Potentially, in a nonimmunocompromised host, with optimal immunogen design, an HIV-1 vaccine can elicit humoral responses that can effectively impact HIV-1 infection (Fig. 1). Several studies, both in nonhuman primates and in humans, are now emerging that characterize the B-cell response after vaccination. 
Detailed studies are needed on how HIV-1 memory and plasma B cells develop in response to different HIV-1 Env vaccination regimens. One study in humans found a direct correlation of Env-specific memory B cells and circulating anti-Env plasma antibodies from the VaxGen VAX004 gp120 trial [50]. A total of $1.79 \%$ of all peripheral IgG memory B cells were specific for HIV-1 consensus B Env gp140 2 weeks after the fourth immunization. This was compared to $0.64 \%$ of all peripheral $\operatorname{IgG}$ memory B cells specific for HIV-1 in chronically infected patients (including patients with and without ART). The half-lives of the anti-Env antibody responses were found to be much shorter compared to the anti-Gag antibody responses (as well as to the antibody response to influenza and tetanus toxoid), suggesting that the anti-Env response is coming from short-lived plasma B cells. In comparison, rapid and robust influenza-specific IgG+ antibody-secreting cell (ASC) response was observed after a booster influenza vaccination [22]. Up to 6\% of peripheral blood B cells were secreting IgG at 7 days after the vaccination. IgG+ memory B cells in this study peaked at approximately $1 \%$ of all B cells at 14 to 21 days after vaccination, which is comparable with the memory B-cell levels reported. However, as much as $70 \%$ of all $\mathrm{IgG}+\mathrm{ASCs}$ purified at 7 days post-vaccination were specific for influenza.

In a study of macaques immunized with Env trimers [51•], total B cells and Env-specific B cells in peripheral blood and in bone marrow were measured. Vaccination induced robust Env-specific B-cell responses and high titers of circulating antibodies. Significant populations of Env-specific IgG+ plasma cells and memory B cells were elicited, although total circulation $\mathrm{IgG}+$ plasma cell or memory B-cell levels remained unaffected. Envspecific IgG+ plasma cells, but not memory B cells, were also detected in the bone marrow after multiple immunizations. Of particular interest was that gp41-specific memory B cells made up for approximately $30 \%$ of the total Env-specific memory B-cell population after the second immunization (first boost), and then diminished to approximately $10 \%$ after the fifth immunization, whereas the gp120 V1/2/3 region-specific memory B-cell population expanded during the same period. Meanwhile, this "shifting" of specificity was not observed in the secreted antibodies, where anti-gp41 antibodies never reached a significant proportion. This study again highlights the importance of examining the B-cell populations, in addition to the circulating antibody responses, to gain insight into how the B cells are stimulated.

\section{Conclusions}

\section{Perturbations in the B-Cell Response to HIV-1: A Significant Hurdle for Vaccine Design?}

Designing the optimal immunogen that can elicit potent broadly neutralizing anti-HIV-1 antibodies is a key goal of HIV-1 vaccine design. Since broadly neutralizing antibodies do not commonly develop in HIV-1-infected patients, and they likely require prolonged antigen stimulation, there are still significant obstacles in our understanding of how to elicit these types of antibodies by nonreplicating immunization strategies. It is unknown if vaccination with HIV-1 proteins and/or vectors that result in HIV-1 antigens being expressed by antigen-presenting cells will be able to completely circumvent the negative effects on B cells that HIV-1 exerts during natural infection and achieve optimal stimulation of the B-cell response.

\section{Perturbations in the B-Cell Response to HIV-1: Treatment for Preservation of HIV-1- Specific B-Cell Responses?}

Early treatment of HIV-1 may serve to preserve important host responses to HIV-1 infection. Recent studies [34•, 37•] that showed a positive impact of early ART on the Bcell response indicate that some of the B-cell perturbations can be minimized by significantly decreasing virus replication in the early stages of disease. Furthermore, a 
passive neutralizing antibody study in infant macaques also suggests that interventions to decrease viral load may preserve B-cell function and enhance the ability of potentially protective antibody responses to develop [38••]. It remains to be seen if, in humans, those treated early will have quicker and better-quality antibody responses than those untreated. It is known that the antibody response is significantly stimulated by virus replication such that many individuals with undetectable viral load can sero-revert. However, there is increasing evidence that some individuals that naturally maintain low levels of virus may have a potent functional antibody response, known as antibody-dependent cellular cytotoxicity [52]. Examining the quality of the plasma and memory B-cell responses in those subjects that naturally control virus replication compared to those who were treated early and followed long term could inform our understanding of how early preservation of the B-cell response impacts long-term immunity.

\section{Acknowledgments}

G.D.T is supported by the Bill and Melinda Gates Foundation (38619), National Institutes of Health (NIH/NIAID) grants: RO1AI052779, AI068618 (HIV Vaccine Trials Network), U19AI067854 (Center for HIV/AIDS Vaccine Immunology), and AI64518 (Duke Center for AIDS Research).

\section{References}

Papers of particular interest, published recently, have been highlighted as:

- Of importance

•• Of major importance

1. Schnittman SM, Lane HC, Higgins SE, et al. Direct polyclonal activation of human B lymphocytes by the acquired immune deficiency syndrome virus. Science. 1986; 233:1084-1086. [PubMed: 3016902]

2. Moir S, Malaspina A, Li Y, et al. B cells of HIV-1-infected patients bind virions through CD21complement interactions and transmit infectious virus to activated T cells. J Exp Med. 2000; 192:637-646. [PubMed: 10974030]

3. Levesque MC, Moody MA, Hwang KK, et al. Polyclonal B cell differentiation and loss of gastrointestinal tract germinal centers in the earliest stages of HIV-1 infection. PLoS Med. 2009; 6:e1000107. [PubMed: 19582166]

4. Herbein G, Gras G, Khan KA, et al. Macrophage signaling in HIV-1 infection. Retrovirology. 2010; 7:34. [PubMed: 20380698]

5. Kuhrt D, Faith SA, Leone A, et al. Evidence of early B-cell dysregulation in simian immunodeficiency virus infection: rapid depletion of naive and memory B-cell subsets with delayed reconstitution of the naive B-cell population. J Virol. 2010; 84:2466-2476. [PubMed: 20032183]

6. Lane HC, Masur H, Edgar LC, et al. Abnormalities of B-cell activation and immunoregulation in patients with the acquired immunodeficiency syndrome. N Engl J Med. 1983; 309:453-458. [PubMed: 6224088]

7. Moir S, Fauci AS. B cells in HIV infection and disease. Nat Rev Immunol. 2009; 9:235-245. [PubMed: 19319142]

8. Cagigi A, Du L, Dang LV, et al. CD27(-) B-cells produce class switched and somatically hypermutated antibodies during chronic HIV-1 infection. PLoS One. 2009; 4:e5427. [PubMed: 19412542]

9. Swingler S, Zhou J, Swingler C, et al. Evidence for a pathogenic determinant in HIV-1 Nef involved in B cell dysfunction in HIV/AIDS. Cell Host Microbe. 2008; 4:63-76. [PubMed: 18621011]

10••. Xu W, Santini PA, Sullivan JS, et al. HIV-1 evades virus-specific IgG2 and IgA responses by targeting systemic and intestinal B cells via long-range intercellular conduits. Nat Immunol. 2009; 10:1008-1017. Xu et al. provide novel information about the function of the HIV-1 
accessory protein, Nef, in modulating the B-cell response through the formation of nanotubulelike structures in infected macrophages. [PubMed: 19648924]

11. Moir S, Fauci AS. Nef, macrophages and B cells: a highway for evasion. Immunol Cell Biol. 2010; 88:1-2. [PubMed: 19859083]

12. Qiao X, He B, Chiu A, et al. Human immunodeficiency virus 1 Nef suppresses CD40-dependent immunoglobulin class switching in bystander B cells. Nat Immunol. 2006; 7:302-310. [PubMed: 16429138]

13. He B, Qiao X, Klasse PJ, et al. HIV-1 envelope triggers polyclonal Ig class switch recombination through a CD40-independent mechanism involving BAFF and C-type lectin receptors. J Immunol. 2006; 176:3931-3941. [PubMed: 16547227]

14. Yates NL, Lucas J, Parks R, et al. 142 HIV Frequently Elicits Mucosal and Plasma Env-Specific IgA With a Rapid Initial Decline In Acute Infection. Journal of AIDS. 2009; 51:Abstract No. 142.

15. Martin G, Roy J, Barat C, et al. Human immunodeficiency virus type 1-associated CD40 ligand transactivates B lymphocytes and promotes infection of CD4+ T cells. J Virol. 2007; 81:58725881. [PubMed: 17392362]

16. Epeldegui M, Thapa DR, De la Cruz J, et al. CD40 ligand (CD154) incorporated into HIV virions induces activation-induced cytidine deaminase (AID) expression in human B lymphocytes. PLoS One. 2010; 5:e11448. [PubMed: 20625427]

17. McElrath MJ, Haynes BF. Induction of Immunity to Human Immunodeficiency Virus Type-1 by Vaccination. Immunity. 2010; 33:542-554. [PubMed: 21029964]

18. Hicar MD, Chen X, Briney B, et al. Pseudovirion particles bearing native HIV envelope trimers facilitate a novel method for generating human neutralizing monoclonal antibodies against HIV. J Acquir Immune Defic Syndr. 2010; 54:223-235. [PubMed: 20531016]

19. Liao L, Chen X, Dixon A, et al. P04-45. Characterization of the plasma cell repertoire in acute HIV-1 infection (AHI). Retrovirology. 2009; 6:P73.

20. Scheid JF, Mouquet H, Feldhahn N, et al. Broad diversity of neutralizing antibodies isolated from memory B cells in HIV-infected individuals. Nature. 2009; 458:636-640. [PubMed: 19287373]

21. Wu X, Yang ZY, Li Y, et al. Rational design of envelope identifies broadly neutralizing human monoclonal antibodies to HIV-1. Science. 2010; 329:856-861. [PubMed: 20616233]

22. Wrammert J, Smith K, Miller J, et al. Rapid cloning of high-affinity human monoclonal antibodies against influenza virus. Nature. 2008; 453:667-671. [PubMed: 18449194]

23. Huang CC, Venturi M, Majeed S, et al. Structural basis of tyrosine sulfation and VH-gene usage in antibodies that recognize the HIV type 1 coreceptor-binding site on gp120. Proc Natl Acad Sci U S A. 2004; 101:2706-2711. [PubMed: 14981267]

24. Pancera M, McLellan JS, Wu X, et al. Crystal structure of PG16 and chimeric dissection with somatically related PG9: structure-function analysis of two quaternary-specific antibodies that effectively neutralize HIV-1. J Virol. 2010; 84:8098-8110. [PubMed: 20538861]

25. Zhou T, Georgiev I, Wu X, et al. Structural basis for broad and potent neutralization of HIV-1 by antibody VRC01. Science. 2010; 329:811-817. [PubMed: 20616231]

26. Xiao X, Chen W, Feng Y, et al. Germline-like predecessors of broadly neutralizing antibodies lack measurable binding to HIV-1 envelope glycoproteins: implications for evasion of immune responses and design of vaccine immunogens. Biochem Biophys Res Commun. 2009; 390:404409. [PubMed: 19748484]

27. Dimitrov DS. Therapeutic antibodies, vaccines and antibodyomes. MAbs. 2010; 2:347-356. [PubMed: 20400863]

28. Ofek G, Tang M, Sambor A, et al. Structure and mechanistic analysis of the anti-human immunodeficiency virus type 1 antibody 2F5 in complex with its gp41 epitope. J Virol. 2004; 78:10724-10737. [PubMed: 15367639]

29. Walker LM, Phogat SK, Chan-Hui PY, et al. Broad and potent neutralizing antibodies from an African donor reveal a new HIV-1 vaccine target. Science. 2009; 326:285-289. [PubMed: 19729618]

30. Cardoso RM, Zwick MB, Stanfield RL, et al. Broadly neutralizing anti-HIV antibody 4E10 recognizes a helical conformation of a highly conserved fusion-associated motif in gp41. Immunity. 2005; 22:163-173. [PubMed: 15723805] 
31. Zhang ZQ, Casimiro DR, Schleif WA, et al. Early depletion of proliferating B cells of germinal center in rapidly progressive simian immunodeficiency virus infection. Virology. 2007; 361:455464. [PubMed: 17223151]

32. Bussmann BM, Reiche S, Bieniek B, et al. Loss of HIV-specific memory B-cells as a potential mechanism for the dysfunction of the humoral immune response against HIV. Virology. 2010; 397:7-13. [PubMed: 19962720]

33. Jacobsen MC, Thiebaut R, Fisher C, et al. Pediatric human immunodeficiency virus infection and circulating IgD+ memory B cells. J Infect Dis. 2008; 198:481-485. [PubMed: 18582200]

34••. Moir S, Buckner CM, Ho J, et al. B cells in early and chronic HIV infection: evidence for preservation of immune function associated with early initiation of antiretroviral therapy. Blood. 2010 Moir et al. compared the preservation of the memory B-cell response in those treated early after HIV-1 infection to treatment of chronic HIV-1 infection, and found that early therapy can serve to minimize damage to the B-cell response to HIV-1 infection. This study has significant implications in deciding when to treat HIV-1-infected subjects.

35. Richard Y, Amiel C, Jeantils V, et al. Changes in blood B cell phenotypes and Epstein-Barr virus load in chronically human immunodeficiency virus-infected patients before and after antiretroviral therapy. J Infect Dis. 2010; 202:1424-1434. [PubMed: 20874514]

36. D’Orsogna LJ, Krueger RG, McKinnon EJ, et al. Circulating memory B-cell subpopulations are affected differently by HIV infection and antiretroviral therapy. Aids. 2007; 21:1747-1752. [PubMed: 17690573]

37•. Pensieroso S, Cagigi A, Palma P, et al. Timing of HAART defines the integrity of memory B cells and the longevity of humoral responses in HIV-1 vertically-infected children. Proc Natl Acad Sci U S A. 2009; 106:7939-7944. This is an important study for understanding the impact of early therapeutic intervention on preservation of memory B-cell responses in vertically HIV-1-infected children. [PubMed: 19416836]

38••. Ng CT, Jaworski JP, Jayaraman P, et al. Passive neutralizing antibody controls SHIV viremia and enhances B cell responses in infant macaques. Nat Med. 2010; 16:1117-1119. Ng et al., through passive infusion of specific antibodies in an SHIV challenge model, demonstrate that an early reduction in virus replication may subvert the destruction of B cells and allow for more protective antibody responses to develop. [PubMed: 20890292]

39. Titanji K, De Milito A, Cagigi A, et al. Loss of memory B cells impairs maintenance of long-term serologic memory during HIV-1 infection. Blood. 2006; 108:1580-1587. [PubMed: 16645169]

40. De Milito A, Morch C, Sonnerborg A, et al. Loss of memory (CD27) B lymphocytes in HIV-1 infection. Aids. 2001; 15:957-964. [PubMed: 11399977]

41. Mattapallil JJ, Letvin NL, Roederer M. T-cell dynamics during acute SIV infection. Aids. 2004; 18:13-23. [PubMed: 15090825]

42. Dykhuizen M, Mitchen JL, Montefiori DC, et al. Determinants of disease in the simian immunodeficiency virus-infected rhesus macaque: characterizing animals with low antibody responses and rapid progression. J Gen Virol. 1998; 79 (Pt 10):2461-2467. [PubMed: 9780052]

43. Peruchon S, Chaoul N, Burelout C, et al. Tissue-specific B-cell dysfunction and generalized memory B-cell loss during acute SIV infection. PLoS One. 2009; 4:e5966. [PubMed: 19543531]

44. Moir S, Malaspina A, Ogwaro KM, et al. HIV-1 induces phenotypic and functional perturbations of B cells in chronically infected individuals. Proc Natl Acad Sci U S A. 2001; 98:10362-10367. [PubMed: 11504927]

45. Moir S, Malaspina A, Pickeral OK, et al. Decreased survival of B cells of HIV-viremic patients mediated by altered expression of receptors of the TNF superfamily. J Exp Med. 2004; 200:587599.

46. Moir S, Ho J, Malaspina A, et al. Evidence for HIV-associated B cell exhaustion in a dysfunctional memory B cell compartment in HIV-infected viremic individuals. J Exp Med. 2008; 205:17971805. [PubMed: 18625747]

47. Nagase H, Agematsu K, Kitano K, et al. Mechanism of hypergammaglobulinemia by HIV infection: circulating memory B-cell reduction with plasmacytosis. Clin Immunol. 2001; 100:250259. [PubMed: 11465955] 
48. 2Cagigi A, Mowafi F, Phuong Dang LV, et al. Altered expression of the receptor-ligand pair CXCR5/CXCL13 in B cells during chronic HIV-1 infection. Blood. 2008; 112:4401-4410. [PubMed: 18780835]

49. Doria-Rose NA, Klein RM, Manion MM, et al. Frequency and phenotype of human immunodeficiency virus envelope-specific B cells from patients with broadly cross-neutralizing antibodies. J Virol. 2009; 83:188-199. [PubMed: 18922865]

50. Bonsignori M, Moody MA, Parks RJ, et al. HIV-1 envelope induces memory B cell responses that correlate with plasma antibody levels after envelope gp120 protein vaccination or HIV-1 infection. J Immunol. 2009; 183:2708-2717. [PubMed: 19625640]

51 . Sundling C, Forsell MN, O'Dell S, et al. Soluble HIV-1 Env trimers in adjuvant elicit potent and diverse functional B cell responses in primates. J Exp Med. 2010; 207:2003-2017. This detailed study profiles the B cell and secreted antibody response following Env trimer immunization in NHPs. [PubMed: 20679401]

52. Lambotte O, Ferrari G, Moog C, et al. Heterogeneous neutralizing antibody and antibodydependent cell cytotoxicity responses in HIV-1 elite controllers. AIDS. 2009; 23:897-906. [PubMed: 19414990] 


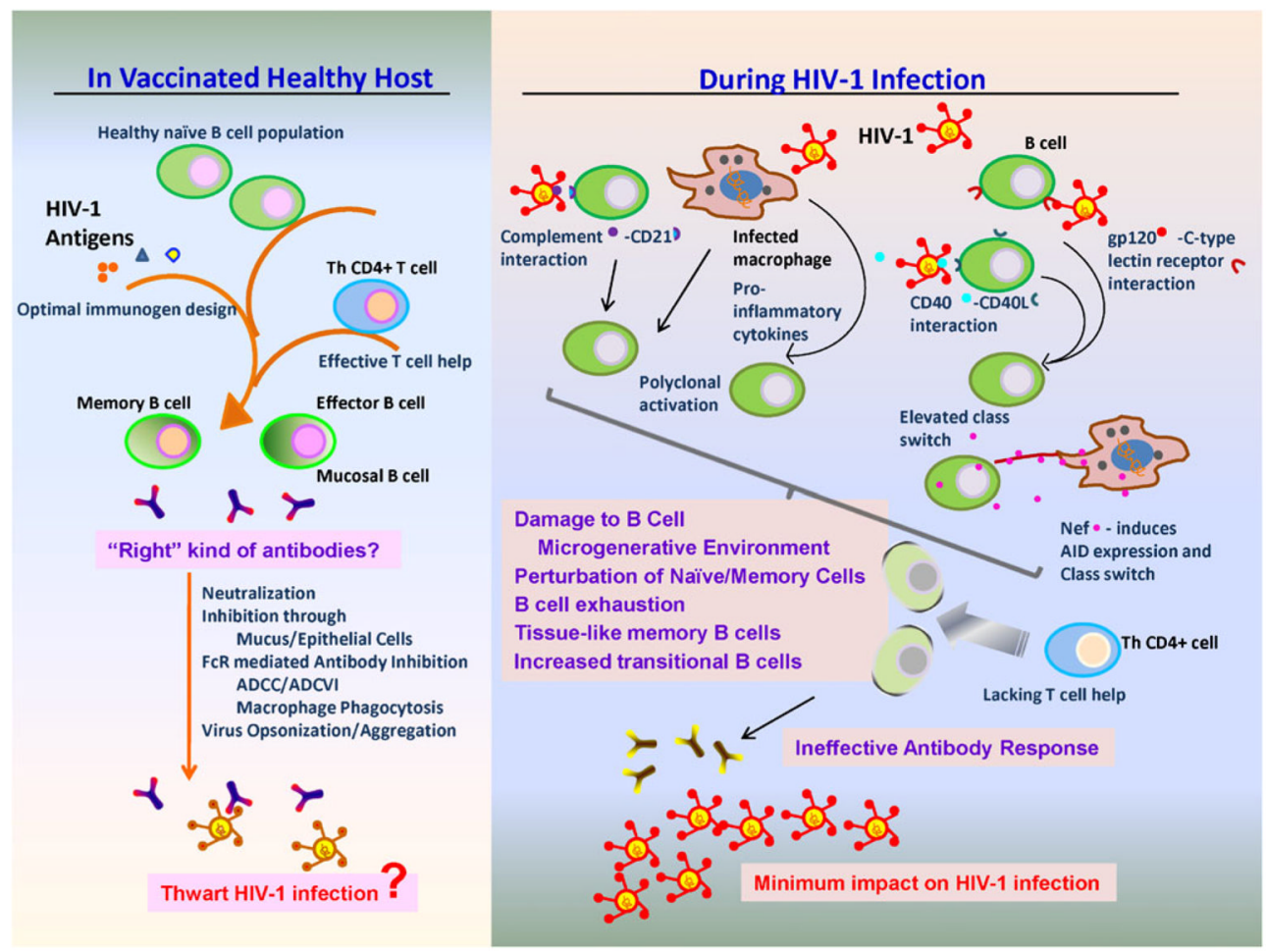

Fig. 1.

Schematic representation of the impaired quality of B-cell responses in HIV-1 infection compared to those responses potentially elicited by vaccination. On the right, HIV-1 infection causes extensive B-cell dysregulation, via a variety of mechanisms, resulting in HIV-1-specific antibody responses incapable of significantly impacting virus infection. On the left, with optimized immunogen design, potent and effective humoral responses may be elicited in a vaccinated healthy host with an uncompromised B-cell response and efficient $\mathrm{T}$ cell help. Antibodies developed in the absence of HIV-1-mediated B-cell dysregulation could then thwart HIV-1 infection via multiple antibody-mediated functions 